\title{
Infected renal cyst: A case report
}

\section{Quiste Renal Infectado: Reporte de Caso}

DDiego Incontri-Abraham, ${ }^{1}$ (DJuan José Juárez-Vignon-Whaley, ${ }^{1}$ (DJorge Magaña.2*

Keywords:

Renal cyst, Bosniak, Pyelonephritis, Urinary

tract infection.

\section{Abstract}

Clinical case description: We present herein an atypical case of a patient diagnosed with an infected renal cyst.

Relevance: Although the presence of solitary renal cysts is more common than expected, they rarely become infected, especially when not associated with a cystic disease.

Clinical implications: Renal cyst diagnosis is mainly made through imaging studies. Infection is rare and normally associated with polycystic kidney disease. Patients do not usually respond well to conventional antibiotic therapy and end up needing drainage and high doses of antibiotics.

Conclusions: There are few case reports of infected renal cyst and they tend to be associated with an underlying pathology, which is important to establish. Even though patients do not usually have a good initial response to antibiotic therapy, they generally have a favorable outcome, with no complications, after drainage and long-term antibiotic administration.

Citación: Incontri-Abraham D., Juárez Vignon-Whaley J.J., Magaña J. Quiste Renal Infectado: Reporte de Caso. Rev. Mex. Urol. 2020;80(6):pp 1-8

\section{Correspondencia:}

*Jorge Magaña.

Dirección: Perugino

No 36. Colonia

Extremadura

Insurgentes. CP 03740,

Benito Juárez, Ciudad

de México. Correo

electrónico:

jorgemaganag@gmail.com

${ }^{1}$ Universidad Anáhuac, Ciudad de México, México

${ }^{2}$ Hospital General de México “Dr. Eduardo Liceaga”, Ciudad de México, México

Recibido: 25 de septiembre de 2019

Aceptado: 14 de septiembre de 2020

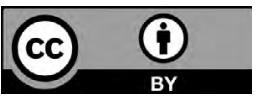




\section{Quiste Renal Infectado: Reporte de Caso. Incontri-Abraham D., et al.}

\section{Resumen}

Descripción del caso clínico: Presentamos el caso atípico de una paciente con diagnóstico de quiste renal infectado.

Relevancia: Aunque la presencia de quistes renales solitarios suele ser más común de lo que parece, que estos se compliquen al grado de infectarse es poco común, aún más si no se asocian a ninguna enfermedad quística.

Implicaciones clínicas: El diagnóstico de un quiste renal es principalmente por medio de estudios de imagen y su infección es rara, normalmente asociada a enfermedad poliquística renal. Los pacientes suelen no responder bien a la antibioticoterapia convencional, por lo que suelen necesitar drenaje y altas dosis de antibióticos.

Conclusiones: Los casos reportados de quiste renal infectado son muy limitados y suelen asociarse a una enfermedad de base, por lo que es importante investigar dicha enfermedad en estos pacientes. Aunque

Palabras clave:

Quiste renal, Bosniak, Pielonefritis, Infección de vías urinarias. los pacientes suelen no responder de primera instancia al tratamiento con antibióticos, después del drenaje y administración de antibióticos a largo plazo suelen presentar un buen pronóstico con ausencia de complicaciones.

\section{Antecedentes}

Los quistes son las lesiones renales benignas más comunes, representando hasta el $70 \%$ de las masas renales, estos pueden ser unilaterales, bilaterales, pueden tener presentación solitaria o múltiple. La incidencia del quiste renal es del $27 \%$ a los 40 años de edad, incrementándose hasta el $61 \%$ a los 80 años. Generalmente se observan en mayores de 50 años y suelen tener un predominio por el sexo masculino. ${ }^{(1-3)}$ Se clasifican como simples o complejos. Los quistes simples generalmente se observan en riñones normales y suelen aparecer durante el envejecimiento. Generalmente son benignos, asintomáticos y sin necesidad de tratamiento. Para diferenciar un quiste simple de uno complejo, existe la clasificación de Bosniak, introducida desde 1986 y donde es necesaria el uso de la tomografía para su evaluación (Tabla 1). ${ }^{(4,5)}$ Es notable el carácter asintomático de estos quistes, por lo que su diagnóstico suele ser debido a un hallazgo por imagen. ${ }^{(2,3)}$ 
Tabla 1. Clasificación de Bosniak

\begin{tabular}{|c|c|}
\hline Categoría I & $\begin{array}{l}\text { Quiste benigno simple } \\
\text { o múltiples } \\
\text { Pared delgada, sin } \\
\text { divisiones (septum) } \\
\text { Ausencia de } \\
\text { calcificaciones } \\
\text { Ausencia de } \\
\text { componentes sólidos } \\
\text { Densidad similar al } \\
\text { agua, sin realce al } \\
\text { contraste } \\
\text { Malignidad: } 0 \%\end{array}$ \\
\hline Categoría II & $\begin{array}{l}<3 \mathrm{~cm} \\
\text { Sin realce al contraste } \\
\text { Pocas divisiones } \\
\text { (septums) } \\
\text { Calcificaciones (gruesas } \\
\text { o pequeñas) } \\
\text { Malignidad: } 0 \%\end{array}$ \\
\hline Categoría IIF & $\begin{array}{l}>3 \mathrm{~cm} \\
\text { Múltiples divisiones } \\
\text { delgadas } \\
\text { Paredes con } \\
\text { calcificaciones } \\
\text { engrosadas/nodulares } \\
\text { Zonas específicas con } \\
\text { realce al contraste } \\
\text { Malignidad: 5\% (F: } \\
\text { "follow-up") }\end{array}$ \\
\hline Categoría III & $\begin{array}{l}\text { Masas quísticas } \\
\text { irregulares } \\
\text { Con realce al contraste } \\
\text { Pared delgada } \\
\text { Varias divisiones } \\
\text { (septums) } \\
\text { Malignidad: } 40-60 \%\end{array}$ \\
\hline Categoría IV & $\begin{array}{l}\text { Características de la } \\
\text { categoría III } \\
\text { Gran realce al contraste } \\
\text { Malignidad: } 85-100 \%\end{array}$ \\
\hline
\end{tabular}

El objetivo de este trabajo es presentar un caso atípico de una paciente con diagnóstico de quiste renal simple infectado.

\section{Caso Clínico}

Presentamos el caso de un paciente femenino de 58 años, con antecedente de diabetes mellitus tipo II e hipertensión arterial sistémica en tratamiento, con diagnóstico previamente conocido de quistes renales bilaterales Bosniak I y sin antecedentes heredofamiliares de importancia. Inicia padecimiento una semana previa con presencia de fiebre cuantificada de $38^{\circ} \mathrm{C}$ y con presencia de dolor de tipo cólico referido en flanco derecho irradiado a región lumbar, así como malestar general. Por lo anterior, la paciente decide acudir al servicio de urgencias del Hospital General de México "Dr. Eduardo Liceaga".

A su llegada, la paciente se encontraba clínica y hemodinámicamente estable, con dificultad para la deambulación, refiriendo dolor de tipo cólico referido en flanco derecho, intensidad 10/10, con irradiación a región lumbar e hipogastrio derecho, con aumento en la intensidad del dolor al toser y moverse o recostarse sobre el mismo lado, sin mejoría de este. A la exploración física dirigida con presencia de Giordano derecho positivo y dolor a la palpación profunda en hipogastrio y flanco derecho. Se realizaron estudios de laboratorio reportando: examen general de orina con parámetros dentro de la normalidad; biometría hemática con leucocitos $11,800 / \mathrm{uL}$, neutrófilos $81 \%$, hemoglobina $12 \mathrm{~g} / \mathrm{dL}$, hematocrito $34 \%$, plaquetas 299,000; química sanguínea con glucosa $348 \mathrm{mg} / \mathrm{dL}$, urea $28 \mathrm{mg} / \mathrm{dL}$, creatinina $0.68 \mathrm{mg} / \mathrm{dL}$, albúmina $3.2 \mathrm{~g} / \mathrm{dL}$.

Dentro de su protocolo de estudio, se realizaron un ultrasonido renal y una tomografía axial computarizada de abdomen y pelvis contrastada (Figura 1). En relación con el riñón derecho, el ultrasonido renal reportó un parén- 
quima heterogéneo debido a una imagen redondeada, de bordes regulares, con dimensiones $7.4 \times 7.1 \times 7.3 \mathrm{~cm}$ en el polo superior y con un volumen aproximado de $338.4 \mathrm{cc}$. Se realizó tomografía de abdomen y pelvis (Figura 2 y 3 ), documentando riñón derecho con presencia de imagen redondeada que desplaza al parénquima renal, bien delimitada, con dimensiones de 8.0 × $8.0 \times 7.0 \mathrm{~cm}$, volumen aproximado de $340.5 \mathrm{cc}$, densidad de 10 Unidades Hounsfield y presencia de gas en su interior, datos compatibles con quiste renal infectado, motivo por el cual fue valorada por servicio de radiología intervencionista, quien realiza drenaje percutáneo guiado por ultrasonido de quiste renal, utilizando como materiales un set de drenaje percutáneo colocándose catéter multipropósito $18 \mathrm{fr}$, asimismo se envió jeringa de 20 cc con muestra del drenaje para cultivo del mismo.

Figura 1. Tomografía contrastada corte axial. Quiste renal en polo superior con presencia de aire en su interior

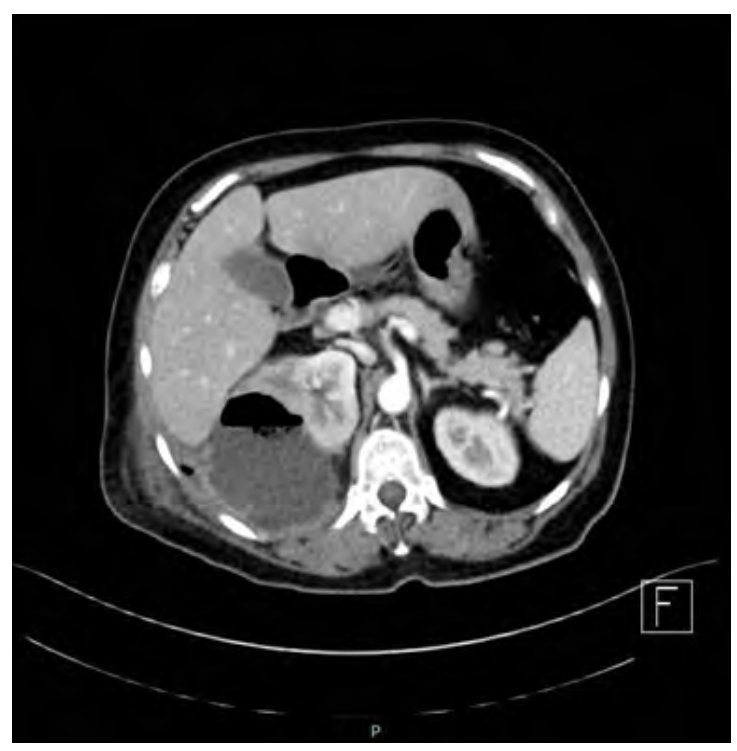

Figura 2. Tomografía contrastada corte coronal. Quiste renal con densidad heterogénea y presencia de gas en su interior

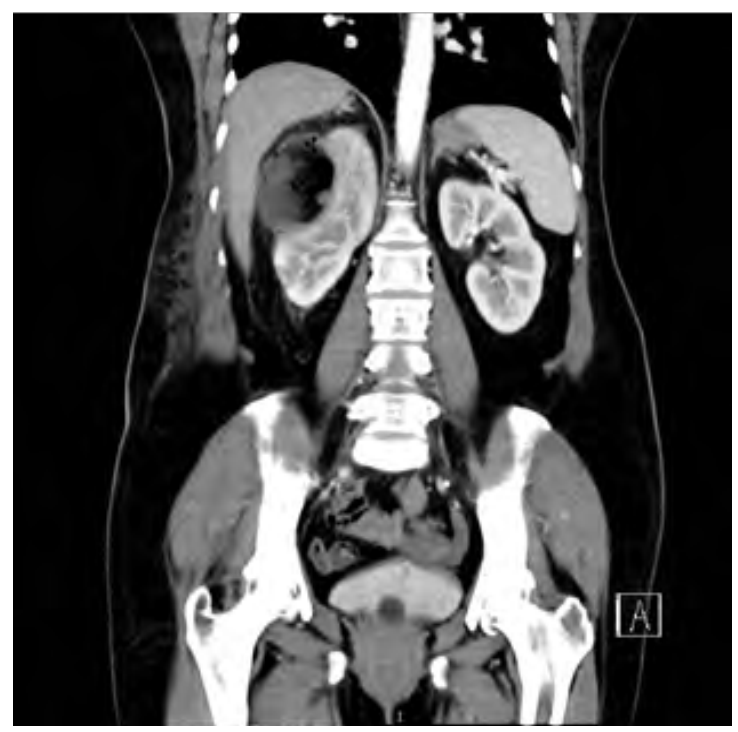

Figura 3. Tomografía contrastada corte coronal. Quiste renal en polo superior con densidad heterogénea

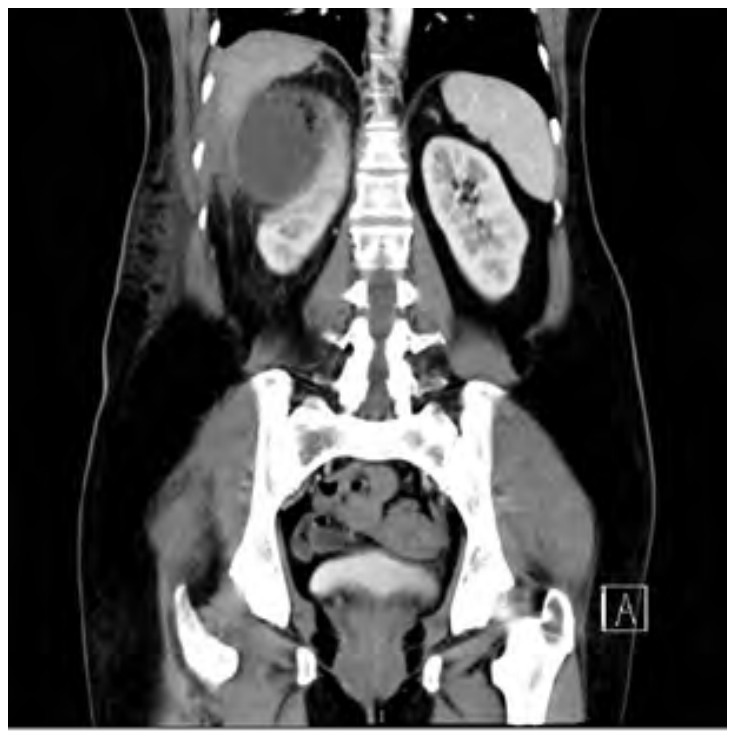

Durante su estancia hospitalaria, la paciente fue tratada con esquema antimicrobiano de amplio espectro indicado por servicio de infectología a base de carbapenémico Ertapenem 1 gramo IV cada 24 horas, permaneciendo con el 
mismo esquema durante 6 días. El reporte de microbiología reportada fue: mas de 100 mil UFC de E. Coli multisensible. Posterior a este reporte se reajusta esquema antimicrobiano dejándose con Ceftriaxona 1 gramo IV cada 12 horas durante 4 días más hasta su egreso. La paciente permaneció hospitalizada durante 10 días, manteniéndose en todo momento clínica y hemodinámicamente estable, con signos vitales dentro de parámetros normales, no se reportaron picos febriles durante su estancia hospitalaria, manteniendo un drenaje promedio de 30cc. Previo a su egreso, se realiza ultrasonido de control, sin evidenciarse colección, por lo que se decide retiro de catéter multipropósito y egreso a domicilio, con seguimiento en consulta externa, con reporte de últimos laboratorios dentro de parámetros normales. (Tabla 2). Debido a que cumplió con esquema antimicrobiano, fue valorada nuevamente por servicio de infectología, concluyendo que la paciente no requirió continuar con esquema antimicrobiano.

Tabla 2. Laboratorios comparativos de ingreso y previos a su egreso

\begin{tabular}{|l|c|c|c|}
\hline \multicolumn{2}{|c|}{ LABORATORIOS INGRESO } & \multicolumn{2}{c|}{ LABORATORIOS EGRESO } \\
\hline Leucocitos & 11,800 & Leucocitos & 6,700 \\
\hline Neutrofilos & $81 \%$ & Neutrofilos & $76 \%$ \\
\hline Hemoglobina & $12 \mathrm{~g} / \mathrm{dL}$ & Hemoglobina & $11 \mathrm{~g} / \mathrm{dL}$ \\
\hline Hematocrito & $34 \%$ & Hematocrito & $33 \%$ \\
\hline Plaquetas & 299,000 & Plaquetas & 245,000 \\
\hline Glucosa & $348 \mathrm{mg} / \mathrm{dL}$ & Glucosa & $155 \mathrm{mg} / \mathrm{dL}$ \\
\hline Urea & $28 \mathrm{mg} / \mathrm{dL}$ & Urea & $30 \mathrm{mg} / \mathrm{dL}$ \\
\hline Creatinina & $0.68 \mathrm{mg} / \mathrm{dL}$ & Creatinina & $0.76 \mathrm{mg} / \mathrm{dL}$ \\
\hline
\end{tabular}

\section{Discusión}

No es frecuente que un quiste renal cause obstrucción y afecte la función. De la misma manera, no suelen asociarse a rupturas, dolor, sangrado o infecciones. En el caso que exponemos, la paciente presentó un quiste renal simple complicado con una infección, una condición poco común de esta entidad. El cuadro clínico de un quiste renal infectado suele ser similar a aquel causado por un absceso renal. La evolución hacia un quiste infectado consiste en la acumulación de material infeccioso dentro del quiste. Para diferenciar un quiste renal infectado de un absceso renal, es preciso observar la respuesta al tratamiento, ya que aquellos con absceso suelen tener una mejor respuesta a an- tibióticos. Por otro lado, y como se presenta en este caso, los pacientes con quistes infectados suelen ser refractarios a la antibioticoterapia. Esto último ocurre debido a que la infección se encuentra encapsulada por el quiste y el antibiótico no logra una adecuada penetrancia. ${ }^{(2,3)}$

En cuanto a la enfermedad renal poliquística, esta constituye un importante diagnóstico diferencial en todo paciente con quistes renales simples, y aún más si se complican con una infección. Esta entidad patológica se hereda de manera autosómica dominante, por lo que los pacientes suelen referir antecedentes familiares de dicha enfermedad. Cabe destacar que esta patología predispone a diversas complicaciones de los quistes renales, tales como: infección quística, hematuria, dolor en flanco, 
insuficiencia renal, entre otras. ${ }^{(3,6)}$ De hecho, los quistes renales infectados representan aproximadamente el 10\% de las causas de hospitalización de pacientes con enfermedad renal poliquística. ${ }^{(7)}$

En caso de que el paciente presente un quiste renal solitario con infección, es importante mencionar que, con base en la literatura evaluada, solamente se han observado alrededor de 8 casos de infección renal quística por bacterias formadoras de gas, en ellas se encuentran: E. Coli, Klebisiella pneumoniae y Proteus. Estas infecciones suelen aparecer en pacientes con enfermedad renal poliquística, sin embargo, en nuestro caso, la paciente niega antecedentes personales y antecedentes familiares de quistes renales. La causa exacta de una infección quística se desconoce, con excepción de la inoculación iatrogénica por medio de una punción. En el caso que presentamos, se sospecha que el origen de la infección del quiste pudo haber sido causada por una infección urinaria previa, o bien, por diseminación hematógena. Como se mencionó anteriormente, estos pacientes no suelen responder bien al tratamiento antibiótico, y el caso presentado no fue la excepción.

Debido a que la paciente no respondió al tratamiento con carbapenémico (ertapenem), fue necesario el tratamiento mediante intervencionismo con colocación de catéter multipropósito para permitir el drenaje del quiste infectado (Figura 4), permitiendo posteriormente adecuada respuesta a manejo antibiótico complementario. Es importante mencionar que, en caso de falla en la respuesta al tratamiento, en ocasiones es necesario recurrir a tratamientos más radicales como nefrectomía parcial. ${ }^{(2,7,8)} \mathrm{Se}$ ha utilizado el drenaje percutáneo con esclerosis para el tratamiento de quistes renales sim- ples, demostrando ser un tratamiento seguro y eficaz, mínimamente invasivo para este tipo de casos. En el caso de quistes renales infectados, el drenaje percutáneo ofrece una opción mínimamente invasiva, altamente resolutiva y segura como opción de tratamiento.

Existen otras alternativas para el drenaje del mismo, la cirugía abierta puede ser una opción válida, sin embargo, al ser un procedimiento invasivo tiene el inconveniente de mayor riesgo de complicaciones asociadas a la intervención, entre ellos lesión a estructuras adyacentes e infección de la herida quirúrgica. Se reserva la cirugía abierta para pacientes con presencia de abscesos renales con involucro a parénquima renal, en los cuales existe la posibilidad de realizar durante el procedimiento de drenaje del absceso, nefrectomía. Existe también la opción de realización de un abordaje laparoscópico, procedimiento de elección para el tratamiento de quistes renales simples, en donde se realiza destechamiento del mismo, sin embargo, tiene la desventaja de ser un procedimiento discretamente más caro que el drenaje percutáneo.

Existen casos reportados en la literatura internacional, en los que, ante la presencia de un quiste renal infectado de menor tamaño (menos de $3 \mathrm{~cm}$ ) se puede indicar únicamente tratamiento antimicrobiano, resolviendo el cuadro, sin embargo esto puede requerir mayor días de estancia hospitalaria. Así mismo, en esta literatura se reporta el manejo quirúrgico abierto, realizando nefrectomía ante la presencia de abscesos renales o quistes renales enfisematosos de gran volumen, no se describen necesidad de reintervención, pero si se describen complicaciones, incluso presentando desenlace fatal en un paciente posterior a la nefrectomía. ${ }^{(2)}$ 
Figura 4. Drenaje percutáneo de quiste renal.

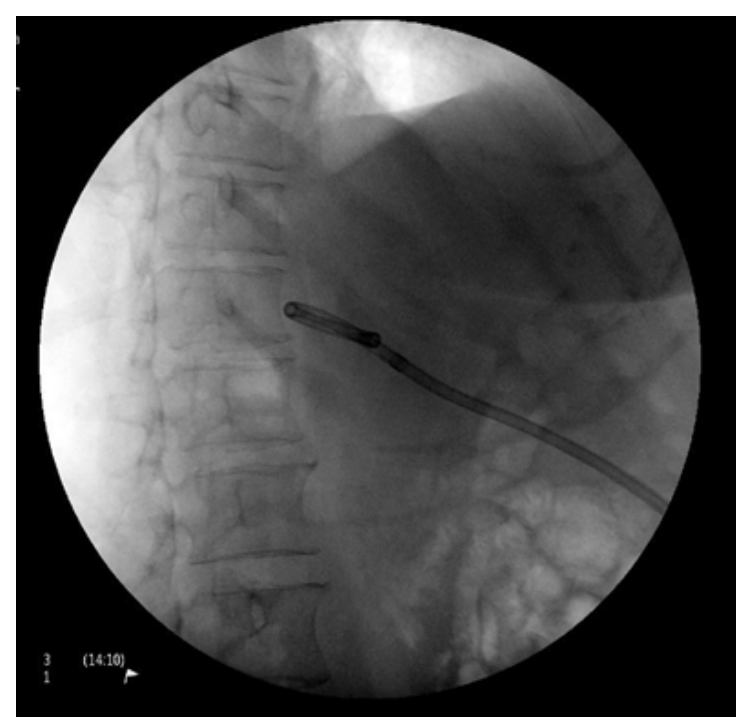

tipo de padecimientos, ya que permite, como lo vimos representado en este caso, una opción de tratamiento segura, eficaz, mínimamente invasiva, resolutiva y con menor días de estancia hospitalaria. Asimismo, permite preservación de las unidades renales y un seguimiento más cuidadoso de los mismos durante su postoperatorio. Comparado con los estudios revisados en la literatura, el drenaje percutáneo de los quistes renales infectados es una opción terapéutica que podrá ofrecerse como tratamiento efectivo, ofreciendo las ventajas previamente mencionadas.

\section{Referencias}

\section{Conclusiones}

Dentro de las complicaciones de los quistes renales, aquellos infectados representan una entidad clínicamente inusual, ocurriendo con mayor frecuencia en pacientes con enfermedad renal poliquística. ${ }^{(7)}$ Sin embargo, ante la presencia de infecciones de repetición o datos compatibles con absceso renal y presencia de un quiste renal, es importante descartar mediante estudios de imagen la presencia de infección quística y, una vez documentada, realizar de forma oportuna un tratamiento que permita el adecuado manejo y resolución del padecimiento. Nuestro paciente es uno de los pocos casos reportados de infección quística. Aunque rara como complicación, esta entidad puede resultar en morbilidad significativa. La correcta identificación del padecimiento en nuestro paciente permitió darle el tratamiento adecuado, y así otorgarle un mejor pronóstico. Es fundamental tener en mente la opción de drenaje percutáneo para el tratamiento de este
1. García-Díaz $\mathbf{M}$, Vega-Castro $\mathrm{R}$, PérezManzanarez VM, Zárate-Morales A, Padilla-Piña J, Gerardo-Osuna $I$, et al. Quiste renal complejo infectado: manejo laparoscópico. Revista Mexicana de Urología. 2015 Mar;75(2):98-100. doi: http://dx.doi. org/10.1016/j.uromx.2015.01.005

2. Ayari Y, Sellami A, Boussaffa H, Taktak T, Ben Rhouma S, Nouira Y. Emphysematous infection of a solitary renal cyst: A case report and literature review. Urol Case Rep. 2018 Sep 28;22:1-2. doi: https://dx.doi.org/10.1016\%2Fj. eucr.2018.09.020

3. Smith AD, Allen BC, Sanyal R, Carson JD, Zhang H, Williams JH, et al. Outcomes and complications related to the management of Bosniak cystic renal lesions. AJR Am J Roentgenol. 2015 May;204(5):W550-556. doi: https://doi.org/10.2214/ajr.14.13149

4. Israel GM, Bosniak MA. An update of the Bosniak renal cyst classification system. Urology. 2005 Sep;66(3):484-8. doi: https:// doi.org/10.1016/j.urology.2005.04.003 


\section{Quiste Renal Infectado: Reporte de Caso. Incontri-Abraham D., et al.}

5. Schoots IG, Zaccai K, Hunink MG, Verhagen PCMS. Bosniak Classification for Complex Renal Cysts Reevaluated: A Systematic Review. J Urol. 2017;198(1):12-21. doi: https://doi. org/10.1016/j.juro.2016.09.160

6. Perdigón-Castañeda GM, Escobar-Hernández N, Pacheco-Gambler C, Ochoa-García A. Punción y esclerosis de quistes renales simples. Anales de Radiología México. 2016 May 15;15(1):9-13.
7. Mohamed AY, Quhal F. Ruptured emphysematous renal cyst. Urol Ann. 2016;8(4):500-2. doi: https://dx.doi. org/10.4103\%2F0974-7796.192091

8. Kennedy-Snodgrass C, Keenan V, Katz DS. Infected Renal Cyst as a Complication of Dropped Gallstones during Laparoscopic Cholecystectomy. Case Rep Gastrointest Med. 2018. doi: https://dx.doi. org/10.1155\%2F2018\%2F2478245 